\title{
Performance of SURF and SIFT Keypoints for the Automated Differentiation of Abnormality in Chest Radiographs
}

\author{
Satyavratan GOVINDARAJAN ${ }^{\mathrm{a}, 1}$ and Ramakrishnan SWAMINATHAN ${ }^{\mathrm{a}}$ \\ ${ }^{a}$ Biomedical Engineering Group, Department of Applied Mechanics \\ Indian Institute of Technology Madras, Chennai, India
}

\begin{abstract}
In this work, automated abnormality detection using keypoint information from Speeded-Up Robust feature (SURF) and Scale Invariant Feature Transform (SIFT) descriptors in chest Radiographic (CR) images is investigated and compared. Computerized image analysis using artificial intelligence is crucial to detect subtle and non-specific alterations of Tuberculosis (TB). For this, the healthy and TB CRs are subjected to lung field segmentation. SURF and SIFT keypoints are extracted from the segmented lung images. Statistical features from keypoints, its scale and orientation are computed. Discrimination of TB from healthy is performed using SVM. Results show that the SURF and SIFT methods are able to extract local keypoint information in CRs. Linear SVM is found to perform better with precision of $88.9 \%$ and AUC of $91 \%$ in TB detection for combined features. Hence, the application of keypoint techniques is found to have clinical relevance in the automated screening of non-specific TB abnormalities using CRs.
\end{abstract}

Keywords. Tuberculosis, Chest radiograph, Speeded-Up Robust feature, Scale Invariant Feature Transform, Keypoint, Support Vector Machine

\section{Introduction}

Early diagnosis of Tuberculosis (TB) can significantly decrease mortality rate globally. Chest radiographs (CR) are widely preferred for this purpose. CR biomarkers such as lung nodular infiltrates and cavitations appear subtle to be detected [1]. Robust local feature extraction methods are necessary to understand the morphological and textural variations in the lung regions. SURF, SIFT keypoint texture descriptors provide localized image patterns and are well-suited for discriminating regions. In this study, performance of SURF and SIFT Keypoints is evaluated for the automated detection of TB in CRs.

\section{Methods}

In this work, 80 (Healthy) and 58 (TB) CRs are obtained from the publicly available Montgomery County TB set. Images are fixed to a resolution of $512 \times 512$ for efficient processing. Segmentation of lung fields is performed using Distance Regularized level

\footnotetext{
${ }^{1}$ Corresponding Author: Satyavratan Govindarajan, Biomedical Engineering Group, Department of Applied Mechanics, Indian Institute of Technology Madras, Chennai, India; E-mail: s.vratan@gmail.com
} 
set (DRLS) [1]. Keypoint features are extracted from lung regions using SURF and SIFT descriptors [2]. Keypoints are examined signifying the changes in the geometry and intensities in different scale-space of normal and TB images. Statistical features such as number of keypoints; mean, standard deviation (SD), minimum, maximum, skewness and kurtosis of scale; mean, SD, minimum, maximum, skewness and kurtosis of orientation are computed to characterize healthy and TB images [3]. Statistically significant features are obtained from Wilcoxon sum rank test. Automated detection of TB is performed with 5-fold cross validation using SVM with different kernels.

\section{Results and Discussion}

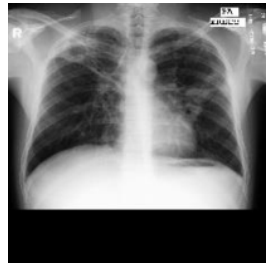

(A)

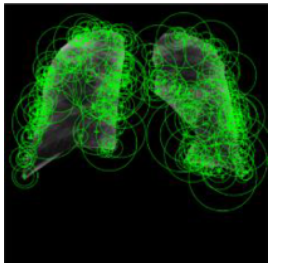

(B)

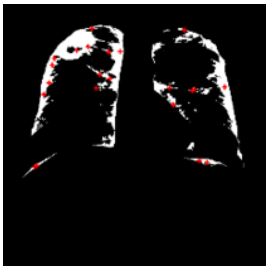

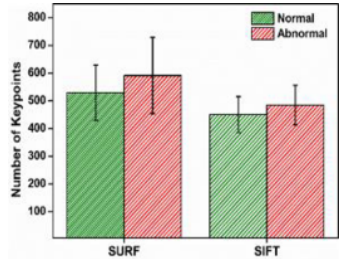

(C)

Figure 1. (A) TB CR image; segmented lungs with (B) SURF \& SIFT keypoints; (C) keypoint variation

From Figure 1 (A), it is seen that the original TB image contain patchy subtle opacities at varied locations in lungs. DRLS method is able to segment lung fields from original CRs. Keypoint features appear to be densely detected at abnormal regions showing local intensity variations on segmented lungs in Figure 1 (B). From barplot, it can be observed that there is a difference between the mean and SD of number of keypoints between healthy and TB images. Five statistical features of SURF and two features of SIFT are found to be significant $(\mathrm{p}<0.05)$.

The classification performance of the extracted features for SVM classifiers is shown in Table 1. Maximum performance with recall, precision and AUC of greater than $82 \%$ is obtained using Linear SVM with combined features as compared to quadratic SVM and individual features. Individually, SURF features performed better in TB detections than SIFT features. The performance of proposed work based on AUC is found to be inline with studies in TB detection using CRs.

Table 1. Performance (\%) of SVM classifiers using significant SURF and SIFT features

\begin{tabular}{ccccccc}
\hline Classifier & \multicolumn{3}{c}{ SVM - Linear kernel } & \multicolumn{2}{c}{ SVM - Quadratic kernel } \\
\hline Measure & SURF & SIFT & SURF+SIFT & SURF & SIFT & SURF+SIFT \\
\hline Recall & 70.7 & 62.1 & $\mathbf{8 2 . 8}$ & 77.6 & 76.3 & 75.9 \\
Precision & 75.9 & 73.5 & $\mathbf{8 8 . 9}$ & 71.4 & 69.7 & 80 \\
AUC & 85 & 80 & $\mathbf{9 1}$ & 86 & 82 & 91 \\
\hline
\end{tabular}

\section{References}

[1] Govindarajan S, Swaminathan R. Analysis of Tuberculosis in Chest Radiographs for Computerized Diagnosis using Bag of Keypoint Features. J Med Syst. 2019 Feb;43:87.

[2] Matos CE, Souza JC, Diniz JO, et al. Diagnosis of breast tissue in mammography images based local feature descriptors. Multimed Tools Appl. 2018 Jul;78:12961-12986.

[3] Kuruvilla J and Gunavathi K. Lung cancer classification using neural networks for CT images. Comput Methods Program Biomed. 2014 Jan; 113(1):202-209. 